\title{
ON ORDERED DIVISION RINGS ${ }^{1}$
}

\author{
PAUL CONRAD
}

In this paper the relation between ordered fields and fields with valuations that was established by Baer [1] and Krull [4] is generalized to ordered division rings. Also some extension theory for ordered division rings is obtained by considering the induced group extensions.

1. Ordered groups. Suppose $\Gamma$ is a simply ordered set and for each $\gamma$ in $\Gamma$ let $R^{\gamma}$ be an additive group. Consider the set $H=H\left(\Gamma, R^{\gamma}\right)$ of all vectors $\left(\cdots, r^{\gamma}, \cdots\right)$ for which $r^{\gamma}$ is in $R^{\gamma}$ and almost every $r^{\gamma}$ is zero (i.e., the set of $\gamma^{\prime}$ s for which $r^{\gamma} \neq 0$ is inversely well ordered). $H$ is a group w.r.t. (with respect to) vector addition, called the $\Gamma$-sum of the $R^{\gamma}$. If the $R^{\gamma}$ are ordered groups (notation $o$-group), then $H$ is an $o$-group w.r.t. the following definition of order: $h$ in $H$ is positive if $h \neq 0$ and the nonzero component with greatest $\gamma$ is positive.

For the rest of this section let $G$ denote an additive o-group. The set $\Gamma_{\theta}$ of all pairs of convex subgroups $G^{\gamma}, G_{\gamma}$ of $G$ where $G^{\gamma}$ covers $G_{\gamma}$ is simply ordered by inclusion, and each $G^{\gamma} / G_{\gamma}$ is $o$-isomorphic to a subgroup of the additive group of real numbers. Thus $G$ determines a $\Gamma_{G}$-sum $H=H\left(\Gamma_{G}, G^{\gamma} / G_{\gamma}\right)$, and $H$ is an abelian $o$-group. The value $V(g)$ of $g \neq 0$ in $G$ is the $\gamma$ in $\Gamma_{G}$ for which $g$ is in $G^{\gamma}$ but not in $G_{\gamma}$. If $a$ and $b$ are elements of $H$ for which $b^{\gamma}=a^{\gamma}$ for all $\gamma>\beta, b^{\gamma}=0$ for all $\gamma \leqq \beta$, and $a^{\beta} \neq 0$, then $b$ is called the $\beta$ th head of $a$.

Lemma 1.1. There exists a 1-1 order and value-preserving mapping $\pi$ of the set $G$ into the set $H$ such that for every $g$ in $G$ :

(a) If $V(g)=\gamma$, then $(g \pi)^{\gamma}=G_{\gamma}+g .0 \pi=0$.

(b) If $a$ is the $\beta$ th head of $g \pi$, then there exists an $h$ in $G$ for which $h \pi=a, V(g-h)=\beta$, and $G_{\beta}+g-h=(g \pi)^{\beta}$.

Proof. There exists a maximal subset $K$ of $G$ for which the lemma holds (apply Zorn's lemma). Suppose that $d$ is in $G$ but not in $K$. To complete the proof it is sufficient to show that $\pi$ can be extended to $\{K, d\}$. Let $V(d)=\delta$. Consider the set $\Delta$ of all elements $x$ in $H$ with $V(x)=\delta, x^{\delta}=G_{\delta}+d$, and such that for every $\beta$ th head $y$ of $x$ there exists an element $h(\beta)$ in $K$ for which $h(\beta) \pi=y, V(d-h(\beta))=\beta$,

Presented to the Society, December 28, 1952; received by the editors July 18, 1953.

1 This work was done under Contract N7-onr-434, Task Order III, Navy Department, The Office of Naval Research, U.S.A. 
and $G_{\beta}+d-h(\beta)=x^{\beta} . \Delta$ is not void since it contains the element $\left(0, \cdots, 0, G_{\delta}+d, 0, \cdots, 0\right)$. We define a partial order over $\Delta$ as follows: $x<y$ if $x$ is a head of $y$. It follows by an application of Zorn's lemma that there exists a maximal element $q$ of $\Delta$. Assume (by way of contradiction) that $q$ is in $K \pi$, and let $k$ be the unique element in $K$ for which $k \pi=q$. Denote the totality of $\gamma$ in $\Gamma_{G}$ for which $q^{\gamma} \neq 0$ by $\Gamma(q)$. For each $\gamma$ in $\Gamma(q)$ there exists an $h(\gamma)$ in $K$ for which $h(\gamma) \pi$ $=$ the $\gamma$ th head of $q$. Moreover $V(d-h(\gamma))=V(k-h(\gamma))=V(h(\gamma)$ $-k)=\gamma, G_{\gamma}+d-h(\gamma)=q^{\gamma}$, and $G_{\gamma}+h(\gamma)-k=-q^{\gamma}$. Thus $\alpha=V(d-k)$ $=V(d-h(\gamma)+h(\gamma)+k)<\gamma$ for all $\gamma$ in $\Gamma(q)$. Let $s$ be the element in $H$ such that $s^{\gamma}=q^{\gamma}$ for all $\gamma \neq \alpha$, and $s^{\alpha}=G_{\alpha}+d-k$. Clearly $q<s$, and $s$ belongs to $\Delta$, a contradiction. Therefore $q$ is not in $K \pi$. Define $d \pi=q . \pi$ is a $1-1$ value-preserving mapping of $\{K, d\}$ into $H$ that satisfies (a) and (b). To complete the proof we must show that $\pi$ preserves order.

Suppose that $d<h$ for $h$ in $K$. If $h=0$ or $V(d)<V(h)$ or $V(d)=V(h)$ and $G_{\delta}+d<G_{\delta}+h$, then $d \pi<h \pi$, since $\pi$ satisfies (a). Assume that $d \equiv h \bmod G_{\delta} ;$ then there exists a $\beta$ in $\Gamma_{G}$ such that $(d \pi)^{\beta} \neq(h \pi)^{\beta}$ and $(d \pi)^{\gamma}=(h \pi)^{\gamma}$ for all $\gamma>\beta$. Let $k$ be the element in $K$ such that $k \pi$ $=\beta$ th head of $h \pi$ (and of $d \pi)$. Then $G_{\beta}+h-k=(h \pi)^{\beta}$ and $G_{\beta}+d-k$ $=(d \pi)^{\beta}$. Thus if $(d \pi)^{\beta}>(h \pi)^{\beta}$, then $G_{\beta}+d-k>G_{\beta}+h-k$, but this means that $d>h$, a contradiction. Therefore $d \pi<h \pi$. By a similar argument it follows that $d>h$ implies $d \pi>h \pi$.

Suppose that $S$ is a subgroup of $G . G$ is an a-extension of $S$ if for every $g>0$ in $G$ there exist an $s>0$ in $S$ and a positive in teger $n$ such that $n s \leqq g \leqq(n+1) s$. $G$ is a c-extension of $S$ if for every $\gamma$ in $\Gamma_{G}$, $G^{\gamma}=G_{\gamma}+\left(S \cap G^{\gamma}\right), G$ is a-closed (c-closed) if it does not admit any proper $a$-extensions ( $c$-extensions). It is clear that $G$ is an $a$-extension of $S$ if and only if $\Gamma_{G}=\Gamma_{S}$ (i.e., $\left(S \cap G^{\gamma}\right) /\left(S \cap G_{\gamma}\right) \neq 0$ for every $\gamma$ in $\left.\Gamma_{G}\right)$. Moreover a $c$-extension is an $a$-extension, hence $a$-closure implies $c$-closure.

CoROLlaRy I. An upper bound for the number of elements in any a-extension of $G$ is $C^{\Delta}$ where $C$ and $\Delta$ are the cardinal numbers of the set of real numbers and the set $\Gamma_{G}$ respectively.

Proof. If $F$ is an $a$-extension of $G$, then there exists a 1-1 correspondence of $F$ into $H\left(\Gamma_{G}, F^{\gamma} / F_{\gamma}\right)$. But there exists an isomorphism of $H\left(\Gamma_{G}, F^{\gamma} / F_{\gamma}\right)$ into $H\left(\Gamma_{G}, R\right)$ where $R=$ reals, and the number of elements in $H\left(\Gamma_{G}, R\right) \leqq C^{\Delta}$. By the usual transfinite arguments we have

Corollary II. There exists an a-closed a-extension and a c-closed c-extension of $G$. 
If $G$ is $c$-closed and every $G^{\gamma} / G_{\gamma}$ is $o$-isomorphic to $R$, then $G$ is $a$-closed. For suppose that $F$ is an $a$-extension of $G$; then $\Gamma_{G}=\Gamma_{F}$ and the 0 -isomorphism $\pi(\gamma)$ that maps $G_{\gamma}+a$ of $G^{\gamma} / G_{\gamma}$ upon $F_{\gamma}+a$ of $F^{\gamma} / F_{\gamma}$ is onto (otherwise we would have an $o$-isomorphism of the additive group of real numbers into a proper subgroup). Thus $F$ is a $c$-extension of $G$, hence $F=G$. If $G$ is abelian, then any two $a$-closed $a$-extensions are equivalent, and $o$-isomorphic to $H\left(\Gamma_{G}, R\right)$. For a proof of this and other results on abelian $o$-groups see Conrad [2].

2. The natural valuation of an ordered division ring. The additive group of a division ring $D$ will be denoted by $+D$, and the multiplicative group by $D^{*} . D$ can be ordered if and only if one of the following conditions is satisfied.

(i) $D^{*}$ has a subgroup of index 2 which is closed w.r.t. addition.

(ii) -1 cannot be represented as a sum of elements of the form $a_{1}^{2} a_{2}^{2} \cdots a_{n}^{2}$, where $a_{i}$ is in $D$ for $i=1,2, \cdots, n$ (Szele [7]).

For the rest of this paper let $D$ denote an ordered division ring. Consider any two elements $\alpha, \beta$ in $\Gamma_{D}=\Gamma+_{D}$, and elements $a, b$ in $D$ such that $V(a)=\alpha$ and $V(b)=\beta$. Define $\alpha+\beta=\gamma$ where $\gamma$ $=V(a b)$. It is easy to prove that $\Gamma_{D}$ is an o-group (w.r.t. the previously defined order in the set $\Gamma_{D}$ ). Moreover $V$ is a valuation of $D$, called the natural valuation of $D .^{2}$ The identity $V(1)$ of $\Gamma_{D}$ will be denoted by $\theta$. Finally we define $V(0)=-\infty$ so that $V(0)<\gamma$, and $\gamma-\infty=-\infty+\gamma=-\infty-\infty=-\infty$ for every $\gamma$ in $\Gamma_{D}$.

Suppose that $R$ is a division ring with valuation $f$ and value group $\Gamma$. For every $\gamma$ in $\Gamma$ we define $R^{\gamma}=R^{\gamma}(f)=\{r$ in $R \mid f(r) \leqq \gamma\}$, and $R_{\gamma}$ $=R_{\gamma}(f)=\{r$ in $R \mid f(r)<\gamma\}$. Clearly $R^{\gamma}$ and $R_{\gamma}$ are abelian groups w.r.t. addition. $R^{\theta}$ is the valuation ring of $f$ in $R$ and $R^{\theta} / R_{\theta}$ is the residue class ring of $R$, w.r.t. $f$.

Lemma 2.1. For every $a, b, c$ in $R$ and $\gamma$ in $\Gamma$ we have: (a) If $f(a)$ $=f(b)=\gamma$, then $a=c b$ where $f(c)=\theta$, and $a \equiv b \bmod R_{\gamma}$ if and only if $c \equiv 1 \bmod R_{\theta}$. (b) There exists a group isomorphism $\pi(\gamma)$ of $R^{\theta} / R_{\theta}$ onto $R^{\gamma} / R_{\gamma}$. (c) The mapping of $h$ in $H\left(\Gamma, R^{\theta} / R_{\theta}\right)$ upon $h \pi$ in $H\left(\Gamma, R^{\gamma} / R_{\gamma}\right)$ where $(h \pi)^{\gamma}=h^{\gamma} \pi(\gamma)$ is an isomorphism.

The proof of (a) follows directly from the definition of a valuation, and (c) is an immediate consequence of (b). Suppose that $x$ is an element of $R$ for which $f(x)=\gamma$. Every element in $R^{\theta} / R_{\theta}$ is of the form $R_{\theta}+c$ where $f(c) \leqq \theta$. Define the mapping $\pi=\pi(x, \gamma):\left(R_{\theta}+c\right) \pi=R_{\gamma}$

2 Here $V(a+b) \leqq \operatorname{Max}[V(a), V(b)]$ instead of the usual condition $V(a+b)$ $\geqq \operatorname{Min}[V(a), V(b)]$ (Schilling $[6$, p. 9]). We prefer this because it makes use of the natural order of $\Gamma_{D}$. 
$+c x$. It can easily be shown that $\pi$ is an isomorphism of $R^{\theta} / R_{\theta}$ onto $R^{\gamma} / R_{\gamma}$. If $R=D$ and $f=V$, then by choosing $x$ positive we have

Corollary. There exists an o-isomorphism of $D^{\theta} / D_{\theta}$ onto $D^{\gamma} / D_{\gamma}$ for every $\gamma$ in $\Gamma_{D}$. The standard arguments show that $D^{\theta} / D_{\theta}$ is o-isomorphic to one and only one subfield of the real numbers which we shall denote by $R_{D}$.

Theorem 2.2. $A$ division ring $R$ can be ordered if and only if (1) there exists a valuation $f$ of $R$ for which $R^{\theta}(f) / R_{\theta}(f)$ can be ordered so that every element of the form $R_{\theta}(f)+a_{1}^{2} \cdots a_{n}^{2}$ is positive, where $a_{i}$ is in $R$ for $i=1,2, \cdots, n$ and $f\left(a_{1}^{2} \cdots a_{n}^{2}\right)=\theta$. Moreover $R$ can be ordered so that $f$ is the natural valuation if and only if (2) the ordering of $R^{\theta}(f) / R_{\theta}(f)$ is Archimedean.

Proof. If $R$ is ordered, then the natural valuation satisfies conditions (1) and (2). Conversely suppose that (1) is satisfied, and let $\Gamma$ be the value group of $f$. Let $S$ be the set of all elements in $R^{*}$ of the form $d_{0} d_{1}^{2} \cdots d_{n}^{2}$, where $d_{i}$ is in $R^{*}$ for $i=0,1, \cdots, n, f\left(d_{0}\right)=\theta$, and $R_{\theta}+d_{0}$ is positive (once again let $R^{\gamma}, R_{\gamma}$ denote $R^{\gamma}(f), R_{\gamma}(f)$ ). By (a) of Lemma 2.1 an element $x$ of $R^{*}$ for which $f(x)=\gamma$ is in $S$ if and only if $x \equiv d_{0} d_{1}^{2} \cdots d_{n}^{2} \bmod R_{\gamma}$ (same conditions on the $d_{i}$ ). If the element $R_{\theta}+s$ in $R^{\theta} / R_{\theta}$ is positive, and $g$ is any element in $R^{*}$, then $R_{\theta}+g^{-1} s g$ is positive. Otherwise $R_{\theta}+g^{-1} s g s=R_{\theta}+\left(g^{-1} s g^{2}\right)^{2}\left(g^{-1}\right)^{2}$ is negative, but this contradicts condition (1). It follows immediately that $S$ is a normal subgroup of $R^{*} . S$ is closed w.r.t. addition. For suppose $d=d_{0} d_{1}^{2} \cdots d_{n}^{2}$ and $c=c_{0} c_{1}^{2} \cdots c_{m}^{2}$ are elements of $S$, and $f(d)=\delta \leqq \gamma=f(c)$. If $\delta<\gamma$, then $d+c \equiv c \bmod R_{\gamma}$, and $f(d+c)=\gamma$. If $\delta=\gamma$, then $d_{0} d_{1}^{2} \cdots d_{n}^{2}=g c_{0} c_{1}^{2} \cdots c_{m}^{2}$, where $f(g)=\theta$. Hence $d_{1}^{2} \cdots$ $d_{n}^{2}\left(c_{m}^{-1}\right)^{2} \cdots\left(c_{1}^{-1}\right)^{2}=d_{0}^{-1} g c_{0}$. Thus by (1) $R_{\theta}+d_{0}^{-1} g c_{0}$ is positive, hence $R_{\theta}+(g+1) c_{0}$ is positive. But $d+c=(g+1) c_{0} c_{1}^{2} \cdots c_{m}^{2}$. Hence in either case $d+c$ is in $S .-1$ is not in $S$. Otherwise $-1=d_{0} d_{1}^{2} \cdots d_{n}^{2}$, hence $R_{\theta}-d_{0}^{-1}=R_{\theta}+d_{1}^{2} \cdots d_{n}^{2}$ is negative, but this contradicts (1).

By Zorn's lemma there exists a maximal normal subgroup $P$ of $R^{*}$ for which $S \leqq P,-1$ is not in $P$, and $P$ is closed w.r.t. addition. Szele [7] proved that $P$ is a subgroup of index 2. Hence $R$ can be ordered (let $P$ be the set of positive elements), and this ordering extends the order of $R^{\theta} / R_{\theta}$. Now suppose that condition (2) is satisfied. To prove that $f$ is the natural valuation it is sufficient to show that $R^{\theta}(f)$ $=R^{\theta}(V) .^{3}$ Thus it is sufficient to show that for every $x>0$ in $R$, $f(x) \leqq \theta$ if and only if $V(x) \leqq \theta$. If $f(x)<\theta$, then $1-m x \equiv 1 \bmod R_{\theta}$ for

3 This is a consequence of Lemma 6, p. 10 in Schilling [6]. The author wishes to thank the referee for indicating this method of proof. 
all integers $m$, so that $1-m x$ is in $S \leqq P$. Thus $m x<1$ for all $m$, hence $V(x)<\theta$. If $f(x)=\theta$, then since $R^{\theta} / R_{\theta}$ is Archimedean there exist positive integers $m$ and $n$ such that $m x+R_{\theta}>1+R_{\theta}$ and $n 1+R_{\theta}>x+R_{\theta}$. Thus $m x>1$ and $n 1>x$, hence $V(x)=\theta$. Finally if $f(x)>\theta$, then $f\left(x^{-1}\right)<\theta$, hence $V\left(x^{-1}\right)<\theta$ and $V(x)>\theta$.

\section{Extension theorems.}

Lemma 3.1. Suppose that $R$ is a division ring with valuation $f$ and value group $\Gamma$. Then there exists an isomorphism $\pi$ of $+R$ into the $\Gamma$-sum $H\left(\Gamma, R^{\gamma}(f) / R_{\gamma}(f)\right)$ [which is isomorphic to $H\left(\Gamma, R^{\theta}(f) / R_{\theta}(f)\right)$ ]. Moreover if $f(a)=\alpha$ for $a$ in $R^{*}$, then $(a \pi)^{\alpha}=R_{\alpha}+a$ and $(a \pi)^{\gamma}=R_{\gamma}$ for all $\gamma>\alpha$.

Proof. Let $P$ be the prime field of $R$. Then ${ }^{+} R, R^{\gamma}(f)$, and $R_{\gamma}(f)$ are abelian operator groups w.r.t. $P$. The pairs $R^{\gamma}(f), R_{\gamma}(f)$ satisfy the definition of a $\Gamma$-group (Conrad [2]). Hence this lemma is a special case of the main embedding theorem.

CoRollary. If $R=D$ and $f=V$, then $\pi$ preserves order and $+D \pi$ is a c-subgroup of $H$.

Suppose that $S$ and $R$ are division rings with valuations $g$ and $f$, and value groups $\Gamma_{o}$ and $\Gamma_{f}$ respectively. Clearly $S$ is an immediate extension of $R$ (Schilling [6, p. 36]) if and only if $S \geqq R, \Gamma_{g}=\Gamma_{f}$, and $S^{\gamma}(g)=S_{\gamma}(g)+R^{\gamma}(f)$ for every $\gamma$ in $\Gamma_{g}$.

THEOREM 3.2. For each division ring $R$ with valuation $f$ there exists a maximal immediate extension.

Proof. If $S$ is an immediate extension of $R$, then $+S$ is isomorphic to a subgroup of the $\Gamma$-sum $H\left(\Gamma_{f}, R^{\theta}(f) / R_{\theta}(f)\right)$. A transfinite argument completes the proof.

An ordered division ring $E$ is an a-extension (m-extension) of $D$ if $E \geqq D$ and $+E$ is an $a$-extension of $+D\left(\Gamma_{E}\right.$ is an $a$-extension of $\left.\Gamma_{D}\right)$.

TheOREM 3.3. $D$ is a-closed if and only if $R_{D}$ is the field of reals and $D$ is maximally complete.

Proof. Assume that $D$ is $a$-closed, then $D$ is maximally complete since an immediate extension is an $a$-extension. Neumann [5] proved that each ordered division ring $G$ admits an $a$-extension $\bar{G}$ that contains a subfield $o$-isomorphic to the reals. Clearly $R_{\bar{G}}=$ reals, hence $R_{D}=$ reals. Conversely assume that $D$ is maximally complete and $R_{D}=$ reals. Then by the corollary to Lemma 3.1 , for any $a$-extension $E$ of $D,+E$ is isomorphic to a $c$-subgroup of the $\Gamma$-sum $H\left(\Gamma_{D}, R_{E}\right)$ 
$=H\left(\Gamma_{D}, R_{D}\right)$. Hence any $a$-extension of $D$ is an immediate extension. Therefore $D$ is $a$-closed.

Corollary. There exists an a-closed a-extension of $D$.

Theorem 3.4. There exists an m-closed m-extension of $D$.

Proof. If $E$ is an $m$-extension of $D$, then $+E$ is isomorphic to a subgroup of $H\left(\Gamma_{E}, R\right)$ where $R=$ reals and $\Gamma_{E}$ is an $a$-extension of $\Gamma_{D}$. By Corollary I of Lemma 1.1 the number of elements in any such $\Gamma_{E}$ is bounded and the bound is determined by $D$. A transfinite argument completes the proof.

REMARKS ON $o$-FIELDS. The $\Gamma$-sum $H=H\left(\Gamma_{D}, R_{D}\right)$ is the additive group of the ordered division ring $\bar{H}$ of all formal power series over $\Gamma_{D}$ with coefficients in $R_{D} . \bar{H}$ is maximally complete. $\bar{H}$ is $a$-closed if and only if $R_{D}=$ reals. $\bar{H}$ is $m$-closed if and only if $\Gamma_{D}$ is $a$-closed and $R_{D}=$ reals. Using Theorem 6, p. 226, Schilling [6], the following well known results can be proved. Each $o$-field $F$ is $o$-isomorphic to a subfield of the field $\bar{H}\left(\Gamma_{F}, R\right)$ where $R=$ reals. $\bar{H}$ is the $a$-closure of the image of $F$ under this isomorphism. Also $F$ is isomorphic to a subfield of the field $\bar{H}(\Gamma, R)$ where $\Gamma$ is the $a$-closure of $\Gamma_{F} . \bar{H}$ is the $m$-closure of the image of $F$ under this isomorphism. Finally, $F$ is $m$-closed if and only if $\Gamma_{F}$ and $F$ are $a$-closed.

\section{BIBLIOGRAPHY}

1. R. Baer, Über nichtarchimedisch geordnete Körper, Sitzungsberichte der Heidelberger Akademie der Wissenschaften vol. 8 (1927).

2. P. Conrad, Embedding theorems for abelian groups with valuations, Amer. J. Math. vol. 75 (1953) pp. 1-29.

3. I. Kaplansky, Maximal fields with valuations, Duke Math. J. vol. 9 (1942) pp. 303-321.

4. W. Krull, Allgemeine Bewertungstheorie, Journal für Mathematik vol. 167 (1932) pp. $160-196$.

5. B. H. Neumann, On ordered division rings, Trans. Amer. Math. Soc. vol. 66 (1949) pp. 202-252.

6. O. F. G. Schilling, The theory of valuations, American Mathematical Society, 1950.

7. T. Szele, On ordered skew fields, Proc. Amer. Math. Soc. vol. 3 (1952) pp. 410413.

Tulane University 